\title{
Clinical efficacy of artemisinin-lumefantrine and status of antifolate drug resistance markers in western Kenya
}

gabriel m kishoyian ( $\sim$ gkishoyian@gmail.com )

Kenya Medical Training College

Eliud N.M. Njagi

Kenyatta University

George 0. Orinda

Kenyatta University

Kevin Thiongo

Kenya Medical Research Institute

Francis T. Kimani

Kenya Medical Research Institute

Damaris Matoke-Muhia

Kenya Medical Research Institute

Research

Keywords: Artemether-lumefantrine, molecular markers, pfk13, malaria, Kenya

Posted Date: December 20th, 2019

DOI: https://doi.org/10.21203/rs.2.19382/v1

License: (9) (i) This work is licensed under a Creative Commons Attribution 4.0 International License. Read Full License 


\section{Abstract}

Background: The development of Plasmodium falciparum resistance to sulfadoxine-pyrimethamine (SP) necessitated its replacement with artemether-lumefantrine (AL) in Kenya. However, the delayed parasite clearance following treatment with artemisinin derivatives has now spread in the Greater Mekong Sub region and may emerge or spread to other malaria endemic regions. Since parasites resistance to drugs is correlated with specific gene mutations, molecular markers associated with antimalarial drugs offer a powerful tool to monitor the emergence and spread of resistance. In this study, we assessed the clinical efficacy of $A L$ following its adoption and the current levels of antifolate drug resistant markers after its ban in Kenyan health facilities.

Methods: We conducted a therapeutic efficacy study between May and November 2015 in Chulaimbo sub-County, Kisumu, Kenya. A total of 76 patients $\geq 6$ and $\leq 60$ months of age with confirmed Plasmodium falciparum mono-infection were enrolled, treated with AL and followed up for a period of 28 days. Study endpoints were adequate clinical and parasitological response (ACPR) on the 28 day and known SP molecular markers of resistance were also determined.

Results: The study showed that $97 \%$ of the participants had cleared parasitemia within 48 hours with an adequate clinical and parasitological response (ACPR) of 100\% clearance on day 3. On Pfdhfr/Pfdhps mutants, N51I was identified in the $P$. falciparum isolates with a prevalence of $94 \%$ while C59R and S108N had $92 \%$ each. The prevalence of mutation at Pfdhps codons A437G and K540E stood at $94 \%$ and $91 \%$ respectively.

Conclusion: AL was found to be highly efficacious since no plasmodium falciparum parasites were observed after day 3 making it a drug of choice in Kenya. Evidence of quintuple mutations frequency is high in the study population threatening the future use of SP.

\section{Introduction}

Malaria is a parasitic infectious disease of global public health concern with sub-Saharan Africa bearing the greatest burden. In 2017, approximately 219 million malaria cases and 446,000 deaths were mostly under-fives and pregnant women from sub-Saharan Africa were reported to occur globally [1]. Malaria parasites include: Plasmodium .falciparum, ovale, malaria, vivax and knowlesi with Plasmodium .falciparum being the most life threatening, and responsible for the majority of morbidity and mortality around the world [1]. For the control and prevention of malaria, World Health Organization (WHO) advocates either vector control (blocking mosquitoes from biting human beings) or chemoprevention (pregnant women, children and other high-risk groups) or specific perspectives for example complex emergencies and elimination[1]. However, the principal mediations recommended by WHO comprise the use of sleeping under net that has been treated (Insecticides Treated Nets) and indoor residual spraying (IRS) [1]. These methods can also be complemented where applicable by use of larval source management [1]. 
Rapidly developing insecticide-resistant vectors to the available insecticides is jeopardizing the effectiveness of these two methods [2]. In addition, larval habitat manipulation and modification, and chemical or biological larviciding has been used in certain countries as a larval source management strategies [2,3]. Despite the success of this strategy to some extend it is not taken as a core strategy to prevent malaria with suggestion that a larval habitat must be well defined for its implementation [3]. An effective vaccine is the cure for complete eradication of the parasite. Regrettably, although the RTS, S vaccine (Mosquirix) which has offered modest protection against malaria in children (Malawi, Ghana and Kenya) may be available in a few years, no highly effective vaccine is on the horizon [4]. Therefore, preventive chemotherapy and case treatment are integrated into the above malaria vector control interventions [3]. Early case detection and prompt treatment is the mainstay to minimize malaria related morbidity and mortality with appropriate use of antimalarial drugs remaining the cornerstone of malaria control [3].

In the early 1940s, chloroquine (CQ) was the drug of choice for the treatment of malaria in many countries having been confirmed as one of the most important anti-malarial drug with quick metabolism, good curative effect including affordable cost [5-8]). Resistance to CQ on P. falciparum isolates were first observed in Thai-Cambodia border in Southeast Asia in 1957 and Venezuela-Combodia border in Northern South America in 1959 and eventually spreading to other countries around the world [9-10]. Over the years, evolvement of drug resistance to the current treatment regimens is a challenge to malaria management. In Africa, resistance to chloroquine (CQ) led to its withdrawal as an antimalarial drug and replaced with SP in the early 1980s[11]. However, soon after, resistance to SP gradually emerged and spread widely throughout sub Saharan Africa) [11]. Malawi was the first country in the continent to cease administration of CQ in malaria chemotherapy in the year 1993 following widespread of treatment failure [12]. In Tanzania, CQ has been used as a first-line treatment of malaria since 1970s but due to high level of resistance, it was replace with SP in 2001. Immediately, resistance to SP emerged and in 2006, AL was adopted and the implementation of the new policy started in 2007 [12-15].

In Kenya, CQ resistant P falciparum was reported in 1977, whereby by 1998 resistant levels had reached $70 \%$ [51]. In 1999, Kenya followed suit as did many sub-Saharan countries on the continent by replacing CQ with SP as the first-line anti-malarial drug [12]. However, widespread reports of declining SP efficacy at the coast [16] and other parts of Kenya [17] soon emerged prompting another first-line antimalarial policy to the currently preferred Coartem TM, an artemether-lumefantrine $(\mathrm{AL})$ combination rolled out in government health facilities since 2006. Recent studies have shown that artemisinin based combination therapy (ACT) is still efficacious and safe for the treatment of uncomplicated falciparum malaria especially in Africa, as well as the current recommended antimalarial drug for the treatment of uncomplicated malaria caused by Plasmodium falciparum $[9,18,19]$.

Of great concern is the unfortunate emergence of resistance to artemisinin which is the cornerstone of ACTs in western Cambodia and subsequently spreading several neighboring countries in Greater Mekong sub region of Southeast Asia in rent years [15, 20-23]. This observation has the potential of reversing the recent gains in malaria control, jeopardizing malaria elimination efforts. The World Health Organization 
(WHO) recommends that malaria-endemic countries should monitor the efficacy of nationally recommended ACT in order to guide national treatment guidelines [9]. Artemisinin-based combinations recommended by WHO includes artemether-lumefantrine (AL), artesunate-amodiaquine ( $A S+A Q)$, artesunate-mefloquine (AS +MQ), artesunate-sulfadoxine/pyrimethamine (AS + SP), and dihydroartemisinin-piperaquine (DP) with AL being the most widely used combination and is currently the first-line antimalarial drug in most malaria endemic countries in the WHO African region $[1,15]$.

However, as with other drugs, the curative effect of ACT has declined gradually along with its use with in vivo P. falciparum susceptibility studies showing reduction over time [24]. Drug efficacy analyses using molecular marker detection is one of the several methods for the surveillance of resistant development and efficacy monitoring [19, 25-26]. The single nucleotide polymorphism (SNPs) at codon 72, 74, 75 and 76 of P. falciparum chloroquine resistance transporter gene (Pfcrt) and 84, 184, 1034, 1042 and 1046 of P. falciparum multidrug resistance 1 gene (Pfmdr1) have been associated with CQR [10]. Polymorphism of the sarcoplasmic/endo-plasmic reticulum Ca2+-ATPase ortholog in Plasmodium (pfSERCA or pfATPase6) has been associated with ART resistance, although the association of SNPs in pfATPase6 with resistance to ART and the underlying mechanism remains to be confirmed [26].

Global searches have identified almost 200 different non-synonymous mutant pfk 13 genotypes with most mutant's occurring at low level prevalence and have uncertain significance [23]. Single-nucleotide polymorphisms (SNPs) in the PF3D7_1343700 Kelch propeller (K13-propeller) have been identified to be a key casual determinant of ART resistance in SE Asia [27]. Several studies carried out from 2009 to 2015 have all implicated K13-propeller gene as being responsible for artemisinin resistance [28-32]. In Africa, limited mutation of K13 propeller were found in Dakar, Uganda, Mali [32-35]. In another study, pfkelch13, a gene located on chromosome 13 of P. falciparum and encoding K13-propeller proteins was identified to play a vital role in conferring ART resistance through whole genome sequencing of ART isolate and in vitro ring-stage survival assay $\left(\mathrm{RSA}_{0}-3 \mathrm{hr}\right)$ [34].

In a study done in 2014, on in vitro delayed response in artemisinin, the findings observed that the kelch 13 gene (pfk13, P. falciparum 3D7_1343700), a locus associated with chromosome 13 showed slow parasites clearance [23]. Likewise, subsequent clinical studies in western Cambodia showed that delayed parasite clearance in clinical trials was associated with several non-synonymous mutations in pfk13 [23]. Specifically, mutations in the distinct propeller region of the kelch 13 protein (codon 441-726) were associated with slow parasites clearance and subsequently with reduced artemisinin susceptibility in vitro studies assessing susceptibility of the ring-stage parasites [23, 35-36]. A central role of pfk13 propeller mutations in mediating ring-stage was confirm by demonstration that parasites that had the mutations showed ring-stage resistance to artemisinin whereas parasites with the wild type allele were sensitive [23].

The resistance of P. falciparum to antimalarial drugs poses a serious threat to the fight against malaria. These outcomes indicate the important of vigilance in malaria-endemic countries for the possible emergence of resistance to artemisinin and partner drugs and treatment failures with ACT. The 
therapeutic efficacy study is the gold standard for monitoring the efficacy of antimalarial medicines in order to inform national malaria treatment policy [37]. Mutations in SP have been associated with pfdhfr and pfdhps respectively. The presence of the quintuple mutant (pfdhfr triple N51I/C59R/S108N plus pfdhps double A437G/K540E) has been associated with SP treatment failure [37]. In this study we aimed at establishing the status of artemether-lumefantrine clinical efficacy after its introduction in Kenya 2006 and the prevalence of molecular markers associated with SP resistance.

\section{Materials And Methods}

\section{Study area}

The study was done in the lake region of Nyanza in Chulaimbo Sub District Hospital in Kisumu rural constituency, Kisumu County, Kenya. The altitude level of the area is 1,131 m above sea level with yearly rainfall between $1200 \mathrm{~mm}$ and $1300 \mathrm{~mm}$. The humidity ranges of $50 \%$ and $68 \%$ with temperature ranging between $20^{\circ} \mathrm{C}$ and $35^{\circ} \mathrm{C}$. Chulaimbo Sub District is a malaria-endemic zone with stable $P$. falciparum transmission [38]. Malaria infection accounts for $38 \%$ of all outpatient visits and $40 \%$ of all admissions mainly children below five years and expectant mothers in the region [5].

\section{Study population}

Children aged 6 to 60 months visiting the outpatient clinic of Chulaimbo sub District Hospital with signs of uncomplicated malaria were recruited for the study in the month of May 2015 to November 2015. Inclusion criteria: children who were residents of the area, with a body weight of $\geq 5 \mathrm{~kg}$, history of fever in the previous 24 hours or fever with temperature $\geq 37.5^{\circ} \mathrm{C}$, infected only with $P$. falciparum and parasitemia in the range of 2,000 to 200,000 asexual parasites per micro liter of blood, no general danger signs of severe and complicated malaria (prostration, breathing difficulties, severe anemia, convulsions and inability to drink or vomiting). Written consent was obtained from the parent or guardian of the child before recruitment into the study. Patients were excluded from the study if they were below 6 months and above 60 months, body weight less than $5 \mathrm{~kg}$ with a history of fever for more than 24 hours with a temperature of above $37.5^{\circ} \mathrm{C}$, multiple infections, any signs describe above of complicated malaria, severe malnutrition (MUAC $<12 \mathrm{~cm}$ ), haemoglobin $<10 \mathrm{gms} / \mathrm{dl}$, infection with other diseases for example pneumonia, on antibiotics and treatment with antimalarial for the last 28 days. In addition, inability to take drug orally, having taken antimalarial chemotherapy in the past two weeks, evidence of liver disease or acute infection other than malaria and unwilling to participate were excluded from the study.

\section{Sampling design}

- This was a cohort study

\section{Sample size determination}


- Sample size determination was calculated using Lwanga and Lemeshaw [39] with 90 patients

- recruited while 76 completing the follow-up to $28^{\text {th }}$

$\bullet$

\section{- Sample collection}

Approximately, $0.05 \mathrm{ml}$ of blood from a finger prick was collected; thick and thin smears were prepared. Pre-treatment (day zero) samples were collected as dried blood spots on 3MM Whatman filter papers, packaged individually into zip lock bags with a desiccant and transported to Malaria laboratory, Centre for Biotechnology and Development, KEMRI, Nairobi for further analysis.

\section{Treatment and follow-up}

Patients were admitted for 3 days for completion of AL dose and assessment of clinical and parasitological outcome as per the WHO guidelines [40]. All children were given orally, supervised by a nurse. Patients were observed for 30 minutes after drug administration and a full dose was readministered if the patient vomited within the stated period. If vomiting persisted, the patient was excluded from the study and treated with parenteral quinine according to national treatment policy. Patients were treated on day 0 (pre-treatment) 1,2,3, 7, 14 and day 28. Clinical and parasitological assessment was done during follow-up visits.

Adverse events, defined as a sign or symptom that was absent at enrollment but manifested during the follow-up period, were assessed by physical examination and by questioning care- givers. Similarly, serious adverse events, defined as conditions that result in death, a life- threatening condition, hospitalization or prolongation of hospitalization, persistent or significant disability or incapacity were monitored.

\section{Microscopic blood examination}

Thick and thin blood smears were prepared and stained using Giemsa stain, examined under the microscope by two independent technologists and parasitemia recorded. For quality control, archived malaria positive control slides were re-examined. The procedure for parasite counting and parasite density calculation was based on the WHO protocol [40].

\section{Treatment outcome classification}

The clinical outcome was based on WHO, [40] as follows: early treatment failure (ETF) which refer to danger signs or severe malaria on day 1,2 or 3, with manifestation of parasitemia or parasitemia on day 2 being higher than on day 0 , regardless of axillary temperature or parasitemia on day 3 with axillary temperature $\geq 37.5^{\circ} \mathrm{C}$ and parasitemia on day $3 \geq 25 \%$ of count on day 0 . Late clinical failure (LCF): this

refers to danger signs or severe malaria with manifestation of parasitemia on any day from day 4 to day 
28 among patients who did not meet any of the criteria of early treatment failure; and presence of parasitemia on any day from day 4 to day 28 with axillary temperature $\geq 37.5^{\circ} \mathrm{C}$ in patients who never met criteria of early treatment failure or late clinical failure. Late parasitological failure (LPF); this refers to parasitemia on any day from day 7 to day 28 with axillary temperature $<37.5^{\circ} \mathrm{C}$ in patients who never met any of the norms of early treatment failure or late clinical failure. Adequate clinical and parasitological response (ACPR); this refers to parasitemia on day 28, irrespective of axillary temperature, in patients who never met criteria of early treatment failure, late clinical failure or late parasitological failure.

\section{Parasite genotyping}

\section{DNA Extraction using chelex method}

DNA extraction was from dried blood spot was done as described previously [41]. Briefly, each dried filter paper was cut into small pieces and soaked in Saponin-phosphate buffered saline (PBS) overnight at $4^{\circ} \mathrm{C}$. This was followed by washing with $1 \times$ PBS and incubated for 30 minutes. The brown solution from the tube was discarded and 50 microliters of the stock $20 \%$ solution and 150 microliters of DNAse free water were added followed by vigorous vortexing. The tubes were then heated at $100^{\circ} \mathrm{C}$ and centrifuged at 10,000 $\mathrm{g}$ for two minutes. Lastly, the supernatant was transferred to a new tube, spun again and a final transfer was done. The DNA product was then stored at $-20^{\circ} \mathrm{C}$

\section{DNA amplification of Pfdhfr and Pfdhps genes}

Extracts from DNA was analyzed for Pfdhfr and Pfdhps gene mutation using MJ Proflex PCR 9700 machine. The PCR amplification followed the protocol described elsewhere [42] with few modifications. PCR reaction tube consisted of $x 10$ PCR buffer (Roche ${ }^{\circledR}$ ) to a final concentration of $x 1,1.5 \mathrm{mM} \mathrm{MgCl}_{2}$, $400 \mu \mathrm{M}$ dNTP mix , 100nM 1 primers each of Pfdhfr F (5'-GAA TGT AAT TCC CTA GAT ATG GAA TAT T-3') (Inqaba) and M4 reverse (5'-TTA ATT TCC CAA GTA AAA CTA TTA GAG CTT C-3') (inqaba), $1.0 \mathrm{U} / \mu \mathrm{L}$ Taq polymerase (Bioline) , $1 \mu \mathrm{L}$ of the DNA template andDNAse water. PCR cycling conditions included: Initial denaturation of $95^{\circ} \mathrm{C}$ for 5 minutes, and 45 cycles of $\left(92^{\circ} \mathrm{C}\right.$ for 30 seconds, annealing $45^{\circ} \mathrm{C}$ for 45 seconds, extension $72^{\circ} \mathrm{C}$ for 45 seconds, 45 cycles), final extension at $72^{\circ} \mathrm{C}$ for 3 minutes then held at $4^{\circ} \mathrm{C}$. The primers M4- $\mathrm{F}$ was used to amplify the region consisting of 326 base pairs containing cys 59 arg and ser108thr.

PCR reaction tube consisted of $x 10$ PCR buffer (Roche ${ }^{\circledR}$ ) to a final concentration of $x 1,1.5 \mathrm{mM} \mathrm{MgCl}{ }_{2}$, 400 $\mu \mathrm{M}$ dNTP, 1 primer each of PfdhfrF1 (AAA TTC TTG ATA AAA CAA CGG AAC CTT TTA) (inqaba) and PfdhfrM3 (TTA ATT TCC CAA GTA AAA CTA TTA GAG CTT C)(inqaba) forward and reverse respectively of $100 \mathrm{nM}, 2.5 \mathrm{U} / \mu \mathrm{L}$ Taq polymerase (Bioline), $0.2 \mu \mathrm{L}$ of DNA product and DNase water. The Polymerase chain reaction cycling conditions included: Initial denaturation of $95^{\circ} \mathrm{C}$ for 5 minutes, and 45 cycles of 
$\left(92^{\circ} \mathrm{C}\right.$ for 30 seconds, annealing $45^{\circ} \mathrm{C}$ for 30 seconds, extension $72^{\circ} \mathrm{C}$ for 45 seconds, 45 cycles), and lastly final extension at $72^{\circ} \mathrm{C}$ for 3 minutes then held at $4^{\circ} \mathrm{C}$.

The initial set of nested reaction used the primers F-M4 (forward and reverse respectively) amplifying the region of 326 base pairs containing Cys 59 arg and ser 108 thr while the subsequent nested reaction used primers M3-F1 to amplify the region consisting of 522 base pairs containing asn51ile and ser108ile. The primers, primers sequences, pair's fragments sizes, and restriction enzymes are shown in Table 1.

\section{Restrictive fragment length polymorphism digests of the Pfdhfr gene}

The restrictive fragment length polymorphism (RFLP) was done as previously described [42] using restrictive endonuclease Tsp5091 (New England Bio labs, Beverly MA) for codons asn51ile of Pfdhfr. Briefly, in $15 \mu \mathrm{L}, 1.5 \mu \mathrm{L}$ of $10 x$ buffer $3,0.15 \mu \mathrm{L}$ of bovine serum antigen, $8 \mu \mathrm{L}$ of amplified DNA, $0.5 \mu \mathrm{L}$ of Tsp5091 restrictive endonuclease was added to $9.85 \mu \mathrm{L}$ of nuclease-free PCR water. This was followed by incubation for 14 hours at $50^{\circ} \mathrm{C}$ with no agitation. Each sample was mixed with $2 \mu \mathrm{L}$ of ABgeneTM $6 \mathrm{x}$ loading dye and loaded onto a 1.5\% agarose gel in TAE buffer (the dye consist of: 15\% (w/v) Ficoll R400, $0.06 \%(\mathrm{w} / \mathrm{v})$ Xylene cyanol FF $30 \mathrm{mM}$ EDTA). The gel was subsequently run for 35 minutes at a voltage of 80 volts on gel electrophoresis tank from Biorad and was viewed on the gel/photo-documentation system for analysis of the results.

\section{Pfdhps gene amplification and digest}

For the outer PCR reaction, the amplification of the Pfdhps gene was done using R/ and R2 (Inqaba) as forward and reverse primers respectively. Each tube consisted x10 PCR buffer (Roche ${ }^{\circledR}$ ) to an absolute concentration of $x 1,25 \mathrm{mM} \mathrm{MgCl}_{2}$ to an absolute concentration of $1.5 \mathrm{mM}, 20 \mathrm{mM}$ dNTP mix to a final concentration of $200 \mu \mathrm{M}, 100 \mathrm{nM}$ primers each of Pfdhps R/R2 (5'AATTGTGTGATTTGTCCACAA-3') and R2 reverse (5'-AACCTAAACGTGCTGTTCAA-3') (inqaba) to a final concentration of $100 \mathrm{nM}, 5 \mathrm{U} / \mu \mathrm{L}$ Taq polymerase (Bioline) to a final concentration of $2.5 \mathrm{U} /$ reaction tube and $3 \mu \mathrm{L}$ of the DNA template. The mixture was topped up to a volume of $30 \mu \mathrm{L}$ with DNAse water. The samples were then loaded onto the thermo cycler set at $95^{\circ} \mathrm{C}$ for initial denaturation for 3 minutes. This was followed by denaturation at $92^{\circ} \mathrm{C}$ for 30 seconds, annealing temperature of $50^{\circ} \mathrm{C}$ for 45 seconds, $72^{\circ} \mathrm{C}$ extension for 1 minute. These conditions were repeated for 30 cycles followed by a final extension for 3 minutes at $72^{\circ} \mathrm{C}$, then halted at $4^{\circ} \mathrm{C}$.

Nested PCR was done using primers K and K/ (Inqaba) as forward and reverse primers respectively. Each reaction tube consisted of x10 PCR buffer to an absolute concentration of x1, $25 \mathrm{mM}$ magnesium chloride to an absolute concentration of $1.5 \mathrm{mM}, 20 \mathrm{mM}$ dNTPs to an absolute concentration of $200 \mu \mathrm{M}$, $10 \mu \mathrm{M}$ each of the nested primers K (5'-TGCTAGTGTTATAGATATAGGATGAGCATC-3') and K/ (5'CTATAACGAGGTATTGCATTTAATGCAAGAA-3') to a final concentration of $100 \mathrm{nM}$ and $5 \mathrm{U} / \mu \mathrm{L}$ Taq polymerase (Roche) to a final concentration of $2.5 \mathrm{U} /$ reaction tube. In addition, $0.2 \mu \mathrm{L}$ of the amplified 
DNA samples thawed on the ice were transferred to each tube. The mixture was topped up to a volume of $60 \mu \mathrm{L}$ with DNAse free PCR water. PCR was then run with the initial denaturation being set at $94^{\circ} \mathrm{C}$, followed by a denaturation temperature of $94^{\circ} \mathrm{C}$ for 30 seconds, annealing temperature of $45^{\circ} \mathrm{C}$ for 1 minute and extension at $72^{\circ} \mathrm{C}$ for 1 minute.

The steps were repeated for 40 cycles and then followed by a final extension at $72^{\circ} \mathrm{C}$ for 3 minutes before halting the reaction at $4^{\circ} \mathrm{C}$. The nested PCR products (K-K/ and R/-R2) were digested using Ava II and Fok I (New England Biolabs, Beverly) for Pfdhps 437 and 540 respectively following procedure described above. The digest products were then electrophoresed on $1.5 \%$ agarose gel at 80 volts for 30 minutes. The primers, primers sequences, pair's fragments sizes and restriction enzymes are shown in Table 1

\section{Restrictive Fragment Length Polymorphism of Pfdhps}

The products of amplification of Pfdhps were subjected to enzyme digestion to detect which of these samples had mutations. The digestion was done according to the instructions of the enzymes supplier. For the target ala437gly, enzyme Ava II was used with the Plasmodium falciparum wild- type strains 3D7 and HB3 acting as a control. For this reaction, $1.5 \mu \mathrm{L}$ of the NEB buffer, $0.4 \mu \mathrm{L}$ of the restricted enzyme Ava II, $1.7 \mu \mathrm{L}$ of nuclease-free water and $11 \mu \mathrm{L}$ of the PCR product were used. The product was then incubated at $37^{\circ} \mathrm{C}$ for 24 hours followed by incubation at $80^{\circ} \mathrm{C}$ for 20 minutes. A $1.5 \%$ agarose gel was prepared and the products run in the presence of a 100bp molecular ladder for 45 minutes. The products were then viewed on a gel documentation system for analysis of the results.

Similarly, digestion was done for the target lys540glu with Fok I acting as the restrictive enzyme. The reaction was carried out according to manufactures instructions with IEC51/86 and 3D7 being used as controls. The digestion was done for a reaction volume of $15 \mu \mathrm{L}$ which included $1.5 \mu \mathrm{L}$ of NEB buffer, 1.7 $\mu \mathrm{L}$ of nuclease-free water, $0.8 \mu \mathrm{L}$ of the restriction enzyme and $11 \mu \mathrm{L}$ of PCR product. The samples were incubated at $37^{\circ} \mathrm{C}$ overnight and the enzyme inactivated at $65^{\circ} \mathrm{C}$ for 20 minutes. A $1.5 \%$ agarose gel was prepared and the products run in the presence of $100 \mathrm{bp}$ molecular ladder for 45 minutes. The products were then viewed on a gel documentation system

\section{Amplification of Pf18sRNA gene}

The amplification of the 18s RNA gene was done to confirm whether the samples were from Plasmodium falciparum species. For this amplification, the primers pairs $18 \mathrm{R}-18 \mathrm{~F}$ were used with the base pairs ( $5^{\prime}$ CTGAGTCGAATGAACTAGCT-3') and (5'-CCATTTTACTCGCAATAACG-3') respectively. For the reaction, $1 \mathrm{x}$ of PCR buffer included $\mathrm{MgCl}_{2}$ ), 400nM, 200nM of primers, $1 \mathrm{U}$ of Taq Polymerase and $1 \mu \mathrm{L}$ of DNA template was used. PCR was then run with the initial denaturation set at $94^{\circ} \mathrm{C}$ for 3 minutes, followed by denaturation at $94^{\circ} \mathrm{C}$ for 1 minute, annealing at $55^{\circ} \mathrm{C}$ for 2 minutes and extension at $72^{\circ} \mathrm{C}$ for 2 minutes. The final extension was set at $72^{\circ} \mathrm{C}$ for 10 minutes with a total of 30 cycles and finally halting the reaction at $4^{\circ} \mathrm{C}$. 


\section{Results}

\section{Demographic characteristics}

A total of 76 Plasmodium falciparum positive samples from children five (5) years and below were collected from the Chulaimbo Sub-District Hospital and analyzed in the current study. All the samples were from children who had no general danger signs of severe and complicated malaria, were symptomatic, mono-infected and positive using microscope. The mean age and standard deviation were 32 months and SD \pm 11.232 while the weight mean in $\mathrm{Kg}$ and the standard deviation was 14.07 and \pm 2.970 respectively. The minimum and maximum age and weight were 12 and 58 months, 8 and 20 respectively. According to gender, males were 43 (56.6\%) while 33 (43.4\%) were females. The geometric mean of microscopic parasite count on day 0 (before treatment) was 120,595 parasites/ $\mu \mathrm{L}$ while the standard deviation was $163,395.1$ (95\% Cl: 82319.4-15887.89). On day 1, the parasite clearance rate had a mean and standard deviation of 3508.93 and 11,783 respectively ( $95 \% \mathrm{Cl}$ : 797.78-6220.67), on day 2, the parasite clearance had a mean and standard deviation of 24 and 170.85 (95\% Cl-15-63.31). Table 2 shows the profile of the children who participated in the study.

\section{Treatment outcomes}

Treatment outcomes were determined according to the classification of World Health Organization as described by 2018) as early treatment failure (ETF), late clinical failure (LCF), late parasitological failure (LPF) and adequate clinical and parasitological response (ACPR). Of the 90 patients recruited in the study, only 76 (84.4\%) patients completed the follow-up after treatment with artemether-lumefantrine while 14 (15.6\%) patients were either lost during follow-up or withdrawn from the study. PCR-corrected, $100 \%$ ACPR was observed among the study population and there was no re-infection. All patients had cleared parasitemia by day-3.

\section{Molecular markers}

The samples collected were analyzed for SP resistant based on the presence of codon 51, 59 and 108 in pfdhfr and codon 437 and 540 mutations in pfdhps genes. The analysis was done using Restrictive Length fragment Polymorphism (RFLP) Tsp5091 enzyme was targeted for codon 51, while 3D7 and DD2 clone were used as controls representing the wild-type and mutant respectively. The wild-type was restricted to $55 \mathrm{bp}, 120 \mathrm{bp}$, and $153 \mathrm{bp}$ while the mutant control was restricted to $55 \mathrm{bp}, 65 \mathrm{bp}, 120 \mathrm{bp}$, and $218 \mathrm{bp}$. This criterion was then used to categorize the samples as either wild-type or mutant. Out of the 76 samples 5(6\%) were classified as wild-type (SP sensitive) and 71(94\%) as mutant meaning they carried the allele that conferred resistance to sulfadoxine-pyrimethamine as shown in Figure 1. The codon 108 was subjected to RLFP using the enzyme Alu 1. Here, the control used was 3D7 as the wild-type control and DD2 as the mutant control. After the digestion, the wild-type was restricted to two fragments of $118 \mathrm{bp}$ and $180 \mathrm{bp}$ while the mutant control did not cut, appearing just like the uncut product. It's on this 
basis that the samples were categorized as either wild-type or mutant. Of the 76 samples, $6(8 \%)$ and 70 (92\%) were classified as wild-type and mutant respectively as explained in Figure 1.

The diverse mutant genotypes related to $d h f p s$ were identified using RFLP for codons 437 and 540 . For codon 437, the enzyme used was Ava II and wild type control used was 3D7 while the mutant control was HB3. Subsequent digestion of the wild-type control gave a fragment of $438 \mathrm{bp}$ while the wild-type gave a fragment of $404 \mathrm{bp}$. Out of the 76 samples, $5(6 \%)$ were classified as wild-type and $71(94 \%)$ as mutants as described in Figure 1. In the same way, for the 540 codons, the enzyme used for digestion was Fokl with IEC513/86 and 3D7 being used as the wild-type and the mutant control respectively. The wild-type control gave a fragment of $105 \mathrm{bp}$ and $138 \mathrm{bp}$ after restrictive digestion. Of the samples digested $7(9 \%)$ were classified as wild-type and 69 (91\%) as mutants as explained in Figure 1.

\section{Discussion}

The effectiveness of antimalarial drugs is a powerful guarantee for malaria control and elimination. Artemisinin has proven to be the most successful anti-malarial drug for the last 10 years and the morbidity and mortality of malaria has declined dramatically due to the use of ACT in highly endemic areas of the world [1]. The emergence and spread of P. falciparum resistance genes to the most commonly available drugs hinders effective control of malaria [8]. Chloroquine (CQ) was widely used in malaria endemic areas but the first case of resistant was documented in Thailand and spread to Africa in 1970s [8]. A cure rate of below the threshold of $90 \%$ calls for a change in treatment policy [43]. In this study we report a high curate with AL which is in line with findings from other countries in Africa such as Congo, Malawi, Uganda Guinea-Bissau, Senegal and Ghana [44-45]. AL is the first-line treatment of choice in most of the malaria- endemic African countries and has remained highly effective a more decade of its use. The current study is similar to another study in Tanzania confirming that this ACT medicine is highly efficacious for the treatment of uncomplicated falciparum malaria [46].

However, the rapid widespread of the parasite resistance to CQ in Africa in the $90 \mathrm{~s}$, led to its official ban as a first-line treatment for uncomplicated malaria in many African countries [47-48]. In Kenya by the Millennium, the prevalence of CQ resistant was $100 \%$ indicating total failure as a first-line anti-malarial drug and was replaced with SP as the first-line antimalarial against uncomplicated malaria in 1998 which was later replaced by AL in 2004 [49]. The outcome of this study confirms a high cure rate and efficacy (100\%ACPR) of the nationally recommended ACTs for the treatment of uncomplicated malaria caused by Plasmodium falciparum since its introduction over a decade ago. This study is similar to an observation in Papua New Guinea which confirms a high efficacy rate [50]. In addition, the ACPR at $100 \%$ in the current study compares well $97.8 \%$ in the PNG study where both studies involved children five years and below. The present study and that in PNG showing high parasite and fever clearance rates in patients infected with P. falciparum confirms the general observation that AL is still efficacious. These studies are similar to a study in Ethiopia, and Burkina Faso which shows high therapeutic cure rate of above $98 \%$ [3]. The high cure rate of $A L$, especially in children less than five years is encouraging, since treatment failure manifest easily in this age group because of low immunity [37]. The observed high efficacy of AL in this 
study is similar to that done in Tanzania and elsewhere in Africa supporting the high efficacy rates of AL despite its use in Africa for over ten years [15].

According to WHO, delayed parasite clearance (slope half-life $>$ 5hour or day 3 positive rate $<10 \%$ ) indicate suspected artemisinin resistance [9]. The current study showed that AL had a fast parasite clearance in terms of parasites clearance constant indicating absence of suspected artemisinin resistance. Similar studies done earlier in Kenya, Uganda, Somalia and Mali and other African countries suggest that artemisinin resistance in Africa has not emerged in African $[15,19]$. The very high ACPR rate of $A L$ in this study indicate excellent therapeutic activity of the antimalarial drug suggesting that $A L$, the most commonly recommended ACT medicine in malaria-endemic countries, remain highly efficacious, despite its use as first-line treatment of uncomplicated falciparum for more than a decade.

Among the Artemisinin-based combination therapies, artesunate-amodiaquine (ASAQ), artesunatesulfadoxine-pyrimethamine (ASSP), dihydroartemisinin-piperaquine (DHA/PPQ), artesunate-mefloquine (ASMQ) and AL are the most commonly recommended ACTs for the treatment of uncomplicated falciparum malaria in African countries. Several recent studies have observed that these ACTs have maintained high efficacy (cure rate $\geq 95 \%$ ) in many of these countries, despite their use for more than a decade [51-53]. However, a lower efficacy ( $<90 \%$ cure rate) of AL was observed from a study in Angola in two different regions in 2013 and 2015. In these studies the administration of the evening dose was not supervised hence no confirmation that this dose was given to the patient. Therefore, it is possible that the decrease cure rate was due to sub-therapeutic doses of the treatment. However, the low cure rate observed in one of the region in Angola in both years might signal consistently reduced AL efficacy in the area [37].

The high efficacious of AL was also observed in northwest Ethiopia where there was the absence of ETF, confirming nonexistence of possible Artemisinin-resistant P. falciparum in the study area. However, in the same study, the absence of ETF and low recurrent malaria (only 1 LCF) indicate highly therapeutic efficacy of both components of AL [3]. Factors such as host immune host nutritional and immune status, initial parasitemia level, pharmacokinetics and pharmacodynamics may influence the therapeutic efficacy of a drug apart from inherent parasite susceptibility [3]. These may result in treatment failure showing low efficacy of otherwise an efficacious drug. At the same time, resistant parasites may be cleared with the help of immune system which may result in exaggerated efficacy of otherwise a less efficacious antimalarial drug [3].

The unfortunate emergence of Artemisinin resistant in Southeast Asia and the China-Myanmar border is a global concern on the treatment of uncomplicated malaria [54]. Therefore, the delay in clearing the parasites tends to compromise the use of Artemisinin for the treatment of malaria, if this occurs; treatment failure is likely to increase [54]. For example clinical efficacy studies in Ethiopia observed that among the study population, there were five treatment failures, (1.1\%) LTF and 4(4.5\%) were LPF while 84 (94.4\%) ACPR confirming that the efficacy of AL for the treatment of uncomplicated falciparum malaria is high thus agreeing with the current study showing a 100\% ACPR. These studies have shown high efficacy 
of $\mathrm{AL}$ in the treatment of uncomplicated malaria and agrees with findings from other east African countries [54]. In Papua New Guinea, therapeutic study conducted across all age groups confirmed high efficacy of AL as the current first-line treatment of uncomplicated malaria [50].The results of the current findings the several studies support the continuation of $A L$ as a first-line treatment for uncomplicated malaria

Several years after the introduction of SP as the first-line antimalarial drug in Kenya, mutant's genotypes continue to increase in frequency. In the present study, the prevalence of mutations at codon N51I, C59R and S108N of the pfdhfr gene and codon A437G and K540E of the Pfdhps gene, which are the major determinants for sulfadoxine-pyrimethamine resistance were highly prevalent with $94 \%, 92 \%, 92 \%, 94 \%$ and $91 \%$ respectively as shown in Fig. 1 . These findings are consistent with the hyper endemic areas in a study carried out in western Kenya which reported a similar increased in the current mutations as $98 \%$, $75 \%, 100 \%, 94 \%$ and $88 \%$ [55]. Similarly, a study carried out in Tanzania (Korogwe-Tanga region) on SP resistant markers observed that the markers remained high even after the replacement of SP as a first-line treatment of uncomplicated malaria in 2006 where the prevalence of codons $51 \mathrm{I}, 59 \mathrm{R}, 108 \mathrm{~N}, 437 \mathrm{G}$, and $540 \mathrm{E}$ was $93 \%, 80 \%, 97.7 \%, 78.6 \%$ and $77.4 \%$ respectively [14]. In addition, a report from Igombe-Mwanza in Tanzania between 2010 and 2011 found the prevalence of 51I, 59R, 108N, 437G, and 540E as 75\%, $82.5 \%, 94.8 \%, 74 \%$ and $69.5 \%$ respectively [14].

However, 14 years of SP withdrawal over $90 \%$ of the isolates in this study have triple mutations of Pfdhfr codon 51, 59 and 108 indicating a high resistance against antifolate in the study area. This study is comparable with an observation in Iran where $95.7 \%$ P. falciparum samples carried the same triple mutations [56]. The high prevalence of triple mutations in the examined isolates in this study is contrary to findings from India, Sri Lanka and Papua New Guinea where the prevalence is low; however the predominant haplotype 51I,59R, and $108 \mathrm{~N}$ are similar to those found in Vietnam, Malaysia, Gabon and Brazil [56]. This may suggest that the Pfdhfr allele has evolved independently due to the drug pressure in geographically different regions of the world. A study in western Kenya from pregnant women between 2008 and 2009 was found to harbor more than $90 \%$ Pfdhps double mutants and more than $80 \%$ of quintuple mutations [57]. In Mozambique, SP resistance quintuple mutation was reported to be above $75 \%$ in 2008 while triple mutation had reached $100 \%$ fixation [14]. These findings are similar to the current study where the triple and double mutants of both Pfdhfr and Pfdhps are above $90 \%$ prevalence.

Therefore, the prevalence of SP resistance P. falciparum parasites reported here mirrors results from other studies using samples from this location [12] and could partly due to SP use in IPTp as the pyrimethamine component of the drug selects for appropriate drug-tolerant variants. In addition, parasite proportions already bear the resistance genotype before its introduction absolved SP use alone as the primary driver for the high mutant frequencies. Also, selection pressure could possibly have been enhanced by a similar-acting antifolate combination drug notably cotrimoxazole [12].

This drug possesses only mild antimalarial potency but is a common prophylactic prescription against opportunistic respiratory tract infections among HIV infections [12] hence may also have perpetuated the 
mutant population. This study has observed that the high prevalence of quintuple mutants and supported by other studies [12, 58-59] confers high-level SP resistance. In Tanzania for example, a study observed that the prevalence of Pfdhps 540E mutation was generally varying in all regions with the lowest in Mtwara $63.9 \%$ and the highest $98 \%$ in Mbeya. Though the southern parts of Tanzania have lower levels of SP resistance, the northwestern part bordering Kenya and Uganda has higher levels of SP resistance [60]. Similarly, an increase of Pfdhps 540E from 33.1\% in 1996 to $99.2 \%$ in 2009 was reported in Kenya [57].

\section{Conclusion}

This study concludes that the cure rate for uncomplicated malaria caused by $\mathrm{P}$ falciparum using artemether-lumefantrine is high. Despite the impressive outcome, intensive surveillance of ACT partner drugs must be conducted regularly. The findings confirm that the fixation of key mutations in the Pfdhfr and Pfdhps genes confers resistance to SP. Therefore, there is urgent need for careful periodic monitoring of the efficacy of these drugs, as treatment failure may occur due to resistant, sub-therapeutic levels that may occur due to non-adherence, or inadequate absorption as well as the persistent of Artemisinin monotherapy in the system and the presence of Artemisinin resistance in neighboring regions. Also, regular molecular monitoring of resistance markers should be done since it's a more cost-effective alternative to the considerable investment necessary to conduct therapeutic studies in more areas of the country.

\section{Abbreviations}

$\mathrm{AL}$

SP

WHO

ETF

LCF

LPF

ACPR

Pfdhfr

Pfdhps

IPTp

ASAQ
Artemether-lumefantrine

Sulfadoxine-pyrimethamine

World Health Organization

Early treatment failure

Late clinical failure

Late parasitological failure

Adequate clinical and parasitological response

Plasmodium falciparum dihydrofolate reductase

Plasmodium falciparum dihydropteroate synthase

Intermittent and preventive treatment programme

Artesunate-amodiaquine

Page $14 / 25$ 
DHA/PPQ Dihydroartemisinin-piperaquine

ASMQ

Artesunate-mefloquine

PCR

Polymerase chain reaction

RFLP

Restriction fragment length polymorphism

\section{Declarations}

\section{Acknowledgement}

My appreciation is for all the patients and their mothers for participating in the study, KEMRI management for allowing me to analyze the samples in the Laboratories especially malaria laboratory staffs.

\section{Author contributions}

GK was involved in study design, data collection, and analysis, interpretation of the results and drafting of the manuscript. KT and GO were involved in data collection and manuscript writing, EN FK, DMM were involved in experimental supervision, interpretation of the results and drafting of the manuscript. All authors read and approved the final manuscript.

\section{Funding}

Funding was partly self-sponsored and partly sponsored by the National Council of Science Technology and Innovation (NACOSTI) Ref No. $5^{\mathrm{TH}}$ calls 2013/2014.

\section{Availability of data and materials}

The raw data is available on request by the editor of publishing journal 


\section{Ethics approval and consent to participate}

The study was accepted by the Scientific and Ethical Unit of the Kenya Medical Research Institute (SSC Protocol no. 2406) and the consent was obtained from the mothers of the participating children

\section{Consent for publication}

Consent was obtained from the mothers that the results can be published provided the names of the children should not appear anywhere.

\section{Competing interest}

The authors declare that they have no competing interests

\section{References}

1. World Health Organization. (WHO). World Malaria Report. 2018

2. World Health Organization. World malaria report. 2016

3. Teklemariam M, Assefa A, Kassa M, Mohammed H, Mamo H. Therapeutic efficacy of artemetherlumefantrine against uncomplicated Plasmodium falciparum malaria in a high transmission area in northwest Ethiopia. Plos one. 2017

4. Cui L, Mharukurwa S, Ndiaye D, Rathod PK, Rosenthal PJ. (2015). Antimalarial drug resistance: Literature review and activities findings of the ICEMR network. American Journal of Tropical Medicine and Hygiene. 2015. 93:3

5. Kiarie WC, Wangai L, Agola E, Kimani FT, Hungu C. Chloroquine sensitivity: Diminish prevalence of chloroquine-resistant gene marker pfcrt-7613 years after cessation of chloroquine use in Msambweni, Kenya. Malar J. 2015; 14:328. 
6. Antony AH, Das S, Parija CS, Padhi S. Sequence analysis of pfcrt and pfmdr1 genes and its association with chloroquine resistance in Southeast India Plasmodium falciparum Genome Data. 2016; 8: 85-90.

7. Lu F, Zhang M, Culleton RL, Xu S, Tang J, Zhou H et al. Return of chloroquine sensitivity to Africa? Surveillance of African Plasmodium falciparum resistance through malaria imported to china. 2017; 10:355.

8. Muhammad HR, Nock HI, Ndams SI, George JB, Deeni Y. Distribution of pfmfr1 and pfcrt chloroquine drug resistance alleles in north-western Nigeria. Malaria World Journal. 2017; 8:15 1-5.

9. World Health Organization. Global report on antimalarial drug efficacy and drug resistance 20002010. Geneva: World Health Organization. 2010

10. Xu C, Wei Q, Yin K, Sun H, Li J, Xiao T et al. Surveillance of antimalarial resistance pfcrt, pfmdr1 amd pfkelch 13 polymorphisms in African Plasmodium falciparum imported to Shangdong Province, China. Scientific Reports. 2018; 8:12951

11. Ngomo JMN, Mawili-Mboumba DP, Mbondo NP, Ella RNN, Akotet MKB. Increased prevalence of mutant allele Pfdhps 437G and Pfdhfr triple mutant in Plasmodium falciparum isolates from rural area of Gabon, three years after the change of malaria treatment policy. Malaria Research and treatment. 2016

12. Okombo J, Kamau AW, Marsh K, Sutherland JC, Ochola-oyier LI. Temporal trends in the prevalence of Plasmodium falciparum drug resistance alleles over two decades of changing antimalarial policy in coastal Kenya. International Journal of Parasitology: Drug and Drug- Resistant. 2014; 4:3 152-163

13. Ministry of Health. National guidelines for malaria diagnosis and treatment. Edited by Mwita A. and Molten F. Dar es Salam, United Republic of Tanzania, 2006. 
14. Matondo, SI, Temba GS, Kavishe AA, KaukiJS, Kalinga A, Van- Zwetselaar M, Reyburn H, Kavishe RA. High levels of sulphadoxine-pyrimethamine resistance Pfdhfr-Pfdhps quintuple mutations: a crosssectional survey of six regions in Tanzania. Malaria Journal. 2014; 13:152.

15. Mandara Cl, Kavishe RA, Gesase S, Mghamba J, Ngadaya E, Mbunji P, Mkude S, Mandike R, Njau R, Mohamed A, Lemnge MM, Warsame M, Ishengoma DS. High efficacy of artemether-lumefantrine for the treatment of uncomplicated falciparum malaria in Muhenza and Kigoma Districts, Tanzania. Malar J. 2018; 17:261

16. Nzila AM, Mberu EK, Sulo J, Dayo H, Winstanley PA, Sibley CH, Watkins WM. Towards an understanding of the mechanism of pyrimethamine-Sulfadoxine resistance in plasmodium falciparum:Genotypung of dihydrofolate reductase and dihydropteroate synthase of Kenyan parasites. Antimicrobial Agents and chemother. 2000. 44:4: 991-996

17. Omar SA, Adagu IS, Gump DW, Ndaru NP, Warhurst DC. Plasmodium falciparum in Kenya: high prevalence of drug resistance-associated polymorphism in hospital admissions with severe malaria in epidemic area. Annals of Tropical Medicine and Parasitology. 2001; 95:7 661-669.

18. World malaria report 2017 - World Health Organization

19. Warsame M, Hassan AM, Hassan AH, Jibril AM, Khim N, Arale AM et al. High therapeutic efficacy of artemether-lumefantrine and dihydroartemisinin-piperaquine for the treatment of uncomplicated falciparum malaria in Somalia. Malar J. 2019; 18:231.

20. Phyo AP, Emergence of artemisinin-resistant on the western border of Thailand: a longitudinal study. Lancet. 2012; 379: 1960-1966

21. Hien TT, Invivo susceptibility of Plasmodium falciparum to artesunate in Binh Phuoc Province, Vietnam. Malar. J. 2012; 11: https://doi.org/10.1186/1475-11-355 
22. Kyaw MP, Nyant MH, Chit K, Aye MM, Aye KH, Aye MM, Lindegardh N, Tarning J, Imwong M, Jacob CJ, Rasmussen C, Perin J, Ringwald P. Reduced susceptibility of Plasmodium falciparum to artesunate in Southern Myanmar. PLOS ONE. 2013

23. Hamphreys G. Association of mutations in the Plasmodium falciparum Kelch13 gene (Pf3D71343700) with parasites clearance rate. WWARN K13 Genotype-Phenotype study group. BMC Medicine. 2019. 17:1

24. Yang C, Zhang H, Zhou R, QianD, Liu Y, Zhao Y, Li S, Xu B. Polymorphism of Plasmodium falciparum K13 propeller gene among migrant workers returning to Henan Province, China from Africa. 2017

25. Barrette A, Ringwald P. Global report on antimalarial drug efficacy and drug resistance 2000- 2010. WHO Press: Geneva. 2010

26. Li J, Chen J, Xie D, Eyi UM, Matesa RA, Obono OMM, Ehapo SC, Yang L, Yang H, Lin M. Limited artemisinin resistance-associated polymorphisms in Plasmodium falciparum K13-propeller and PfATPase6 gene isolated from Bioko Island, Equatorial Guinea. International Journal of Parasitology: Drug and drug resistance. 2016; 6: 54-59

27. Mok S, Ashley EA, Ferreira PE, Zhu L, Lin Z, Yeo T et al. Drug resistance. Population transcriptomics of human malaria parasites reveals the mechanism of artemisinin. Science. 2015; 347 431-435

28. Talunzic E, Okoth SA, Congpuong K, Plusinski MM, Morton L, Goldman IF, Kachur PS, Wongsrichanalai C, Satimai W, Barnwell JW, Udhayakumar V. Selection and spread of artemisininresistant alleles in Thailand prior to the global artemisinin resistance containment campaign. PloS Pathog. 2015; 11

29. Tun KM, Imwong M, Lwin KM, Win AA, Hlaing TM, Hlaing T et al. Spread of artemisinin-resistant Plasmodium falciparum in Myanmar: a cross-sectional survey of the K13 molecular marker. Lancet. Infectious Disease. 2015; 15 415-421

30. Wang Z, Wang Y, Cabrera M, Zhang Y, Gupta B, Wu Y et al. Artemisinin resistance at the ChinaMyanmar border and association with mutations in the k13 propeller gene. Antimicrob. Agents Chemother. 2015; 59 6952-6959. 
31. Cooper RA, Conrad MD, Watson QD, Huezo SJ, Ninsiima H, Tumwebaze P et al. Lack of artemisinin resistance in Plasmodium falciparum in Uganda based on parasitological and molecular assays. Antimicrob. Agents Chemother. 2015; 59 5061-5064.

32. Outtara A, Kone A, Adams M, Fofana B, Maiga AW, Hampton S et al. 2015. Polymorphism in the K13 propeller gene in artemisinin-susceptiple Plasmodium falciparum parasites from Bougoula-Hameau and Bandiagara, Mali. Am. J. Trop. Med. Hyg. 92 1202-1206

33. Torentino-madamet M, Fall B, Benoit N, Camara C, Amalvic R, Fall M et al. Limited polymorphism in Plasmodium falciparum isolates from Dakar Senegal in 2012-2013. Malar J. 2014; 13472

34. Kamau E, Campino S, Amenga-Etego L, Drury E, Ishengoma D, Johnson K et al. K13 propeller polymorphism in Plasmodium falciparum parasites from sub-Saharan Africa. Journal of Infectious disease. 2015; 211 1352-1355.

35. Ariey F, Witkowski B, Amaratunga C, Beghain J, Langlois AC, Khim N et al. A molecular marker of artemisinin-resistant Plasmodium falciparum malaria. Nature 2014; 505(7481): 50-55

36. Chotivanich K, Tripura R, Das D, Yi P, Day NPJ, Pukrittayakamee S et al. Laboratory detection of artemisinin-resistant Plasmodium falciparum. Antimicrobs Agents Chemother. 2014; 58(6): 31573161

37. Smith SJ, Kamara AYR, Sahr F, Samai M, Swaray AS, Menard D, Warsame M. Efficacy of artemisininbased combination therapies and prevalence of molecular markers associated with artemisinin and sulfadoxine-pyrimethamine resistant in Kenya. Acta Tropica. 2018; 185 363-370

38. Kenya Malaria Indicator Survey. 2015

39. Langa and Lemeshow. Sample size determination in health statistics. A practical manual. 1991. 
40. World Malaria Report 2009 - WHO. https://www.who.int > malaria

41. Warhurst DC, Awad el karim FM, Miles MA. Simplified preparation of malaria blood samples for polymerase chain reaction. Lancet 1991; 337 303-304.

42. Ranford-Cartwright LC, Johnston KL, Abdel-Muhsin AM, Khan BK, Babiker HA. Critical comparison of molecular genotyping methods for detection of drug resistant Plasmodium falciparum. Trans. R. Soc. Trop. Med. Hyg. 2002; 96: 568-572.

43. World Health Organization. 2010b. Status report on artemisinin and ACT resistant. http://www.moh.gov.cn/mohbgt/s10788/201005/47529.shtml.

44. Plucinski M, Mateuzs M, Dimbu PR, Fortes F. Efficacy of artemether-lumefantrine, artesunateamodiaquine, and dihydroartemisinin-piperaquine for treatment of uncomplicated plasmodium falciparum malaria in Angola. Malar Journal 2015; 16:62.

45. Ogutu BR, Onyango KO, Koskei N, Omondi EK, Ongecha JM, Otieno GA et al. Efficacy and safety of artemisinin-lumefantrine and dihydroartemisinin-piperaquine in the treatment of uncomplicated Plasmodium falciparum malaria in Kenyan children aged less than five years: results of an openlabel, randomized, single-centered study. Malar J. 2014; 13:33

1. Kakolwa MA, Mahende MK, Ishengoma D, Mandara Cl, Ngasal B, Kamugisha E et al. Efficacy and safety of artemisisnin-based combination therapy, and molecular markers for artemisinin and piperaquine resistance in Mainland Tanzania. Malar J. 2018; 17:369

47. World Health Organization. Position Paper of WHO Roll Back Malaria Department on malaria treatment policy. Geneva: WHO. 2004

48. World Health Organization. The World Health Report 2006-working together for health. 2006

49. World malaria report 2013. Geneva: World Health Organization; 2013

50. Tavul L, Hetzel MW, Teliki A, Walsh D, Kiniboro B, Rare L et al. Efficacy of artemether-lumefantrine and dihydroartemisinin-piperaquine for the treatment of uncomplicated malaria in Papua New Guinea. Malar J. 2018; 17:350 
51. Abuaku BK, Mensah BA, Ofori MF, Myers-Hansen J, Derkyi-Kwarteng AN, Essilfie F. Efficacy of artesunate/amodiaquine in the treatment of uncomplicated malaria among children in Ghana. Am. J. Trop. Med. Hyg. 2017; 97 690-695

52. De Wit M, Funk AL, Moussally K, Nkuba DA, Siddiqui R, Bil K. In vivo efficacy of artesunateamodiaquine and artesunate-lumefantrine for the treatment of uncomplicated falciparum malaria: an open randomized, non inferiority clinical trial in South Kivu, Democratic Rebublic of Congo. Malar J. 2016; 15455

53. Dorkenoo AM, Yehadji D, Agbo YM, Layibo Y, Agbeko F, Adjeloh P. Therapeutic efficacy trial of artemisinin based combination for the treatment of uncomplicated malaria and investigation of mutation in K13 propeller dormain in Togo. 2012-2013. Malar J. 2016; 15331

54. Mekonnen KS, Medhin G, Berhe N, Clouse RM, Aseffa A. Efficacy of artemether-lumefantrine therapy for the treatment of uncomplicated Plasmodium falciparum malaria in Suthwestern Ethiopia. Malar J. $2015 ; 14: 317$

55. Zhong D, Afrane Y, Githeko A, Cui L, Menge DM, Yan G. Molecular epidemiology of drug-resistant malaria in western Kenya. BMC Infectious Diseases. 2008; 8:105

56. Zakeri S, Farahani MS, Afsharpad M, Salehi M, Raeisi A, Djadid ND. High prevalence of the $437 G$ mutation associated with sulfadoxine resistance among Plasmodium falciparum clinical isolates from Iran, three years after the introduction of sulfadoxine-pyrimethamine. International Journal of Infectious Diseases. 2009; 14 123-128.

57. Juma DW, Omondi AA, Ingasia L, Opot B, Cheruiyot A, Yeda R et al. Trends in resistance codons in Plasmodium falciparum dihydrofolate reductase and dihydropteroate synthatase genes in Kenyan parasites from 2008-2012 Malar Journal. 2014; 13:250

58. Heinberg A, and Kirkman L. The molecular basis of antifolate resistance in Plasmodium falciparum: looking beyond point mutations. Annals of the New York Academy of Sciences. 2015; 1342:1 10-18 
59. Alifrangis M, Nag S, and Roper C. Independent origin of Plasmodium falciparum Antifolate superresistance in Uganda, Tanzania and Ethiopia. Emerging Infectious Diseases. 2014

60. Kavishe RA, Kaaya RD, Nag S, Camilla K, Notland JG, Kavishe AA et al. Molecular monitoring of Plasmodium falciparum super-resistance to sulfadoxine-pyrimethamine in Tanzania. Malaria Journal. 2016; 15335

\section{Tables}

Table 1: Primers, primers sequences, pairs fragments sizes and restriction enzymes used in detection of gene polymorphisms in Pfdhfr and Pfdhps.

\begin{tabular}{|c|c|c|c|c|c|c|}
\hline ne & Primers & Primers sequences & $\begin{array}{l}\text { Size } \\
\text { (bp) }\end{array}$ & $\begin{array}{l}\text { Restriction } \\
\text { enzyme }\end{array}$ & $\begin{array}{l}\text { Fragment length } \\
\text { (wild type) }\end{array}$ & $\begin{array}{l}\text { Fragment length } \\
\text { (mutant) }\end{array}$ \\
\hline $\begin{array}{l}\text { lhfr } \\
\text { tter) }\end{array}$ & $\begin{array}{l}\text { F } \\
\text { M4 }\end{array}$ & $\begin{array}{l}\text { 5'-GAATGTAATTCCCTAGATATGGAATATT-3' } \\
\text { 5-'TTAATTTCCCAAGTAAAACTATTAGAGCTTC- } \\
\text { 3' }\end{array}$ & 326 & Alul & 189,166 & No digestion \\
\hline sted) & $\begin{array}{l}\text { M3 } \\
\text { F1 }\end{array}$ & $\begin{array}{l}\text { TTAATTTCCCAAGTAAAACTATTAGAGCTTC } \\
\text { 5'-AAATTCTTGATAAAACAACGGAACCTTTTA- } \\
\text { 3' }\end{array}$ & 522 & Tsp5091 & $55,65,120,153$ & $5565,120,218$ \\
\hline $\begin{array}{l}\text { lhps } \\
\text { tter) }\end{array}$ & $\begin{array}{l}\mathrm{R} / \\
\mathrm{R} 2\end{array}$ & $\begin{array}{l}\text { 5'AATTGTGTGATTTGTCCACAA-3' } \\
\text { 5'-AACCTAAACGTGCTGTTCAA-3' }\end{array}$ & 438 & Fok I & 438 & $33,85,320$ \\
\hline sted) & $\mathrm{K}$ & $\begin{array}{l}\text { 5’TGCTAGTGTTATAGATATAGGATGAGCATC- } \\
\text { 3' } \\
\text { 5'- } \\
\text { CTATAACGAGGTATTGCATTTAATGCAAGAA-3' }\end{array}$ & 438 & Ava II & 438 & 405 \\
\hline
\end{tabular}


Table 2: Profile of children on therapeutic in the study population

\begin{tabular}{|c|c|}
\hline Characteristics & Artemether-lumefantrine \\
\hline & $\mathrm{n}=76$ \\
\hline Male n (\%) & $43(56.6 \%)$ \\
\hline Female's n (\%) & $33(43.4 \%)$ \\
\hline Mean age in months & 32 \\
\hline Standard deviation & 11.232 \\
\hline Range in age & $12-58$ \\
\hline Mean weight in $\mathrm{Kg}$ & 14.07 \\
\hline Standard deviation & 2.970 \\
\hline Range & $8-20$ \\
\hline Temperature mean on day 0 & 38.12 \\
\hline Standard deviation & 1.081 \\
\hline Parasitemia (per $\mu \mathrm{L}$ ) on day 0 geometric mean & 120,595 \\
\hline Range & $880-832,000$ \\
\hline
\end{tabular}

\section{Figures}




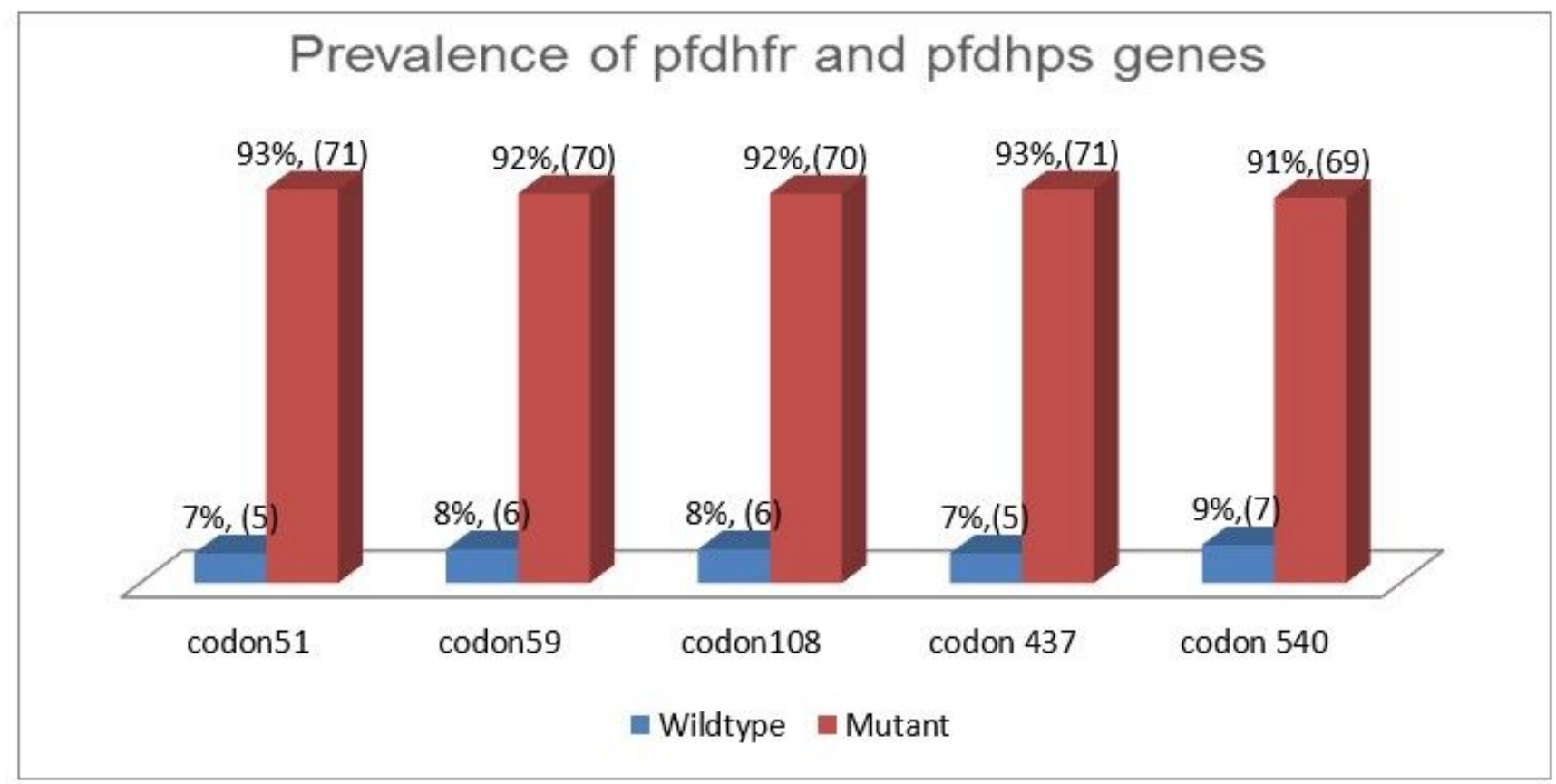

Figure 1

Prevalence of Pfdhfr and Pfdhps wild type and mutant genotypes generated from the study 